\title{
Communication
}

\section{Generation of glyco-engineered BY2 cell lines with decreased expression of plant-specific glycoepitopes}

\author{
Bo-jiao Yin ${ }^{1,2^{*}}$, Ting Gao ${ }^{1 *}$, Nuo-yan Zheng ${ }^{1,2}$, Yin Li ${ }^{2}$, San-yuan Tang1, Li-ming Liang ${ }^{2}$, Qi XIE ${ }^{1 凶}$ \\ ${ }^{1}$ State Key Laboratory of Plant Genomics, National Center for Plant Gene Research, Institute of Genetics and Developmental \\ Biology, Chinese Academy of Sciences, Datun Road, Beijing 100101, China \\ 2 State Key Laboratory of Biocontrol, School of Life Sciences, Sun Yat-sen (Zhongshan) University, 135 Xingang Road W, \\ Guangzhou 510275, China
}

$\triangle$ Correspondence: qxie@genetics.ac.cn

Received December 22, 2010 Accepted January 6, 2011

\begin{abstract}
Plants are known to be efficient hosts for the production of mammalian therapeutic proteins. However, plants produce complex $\mathrm{N}$-glycans bearing $\beta 1,2-x y l o s e$ and core $\alpha 1,3$-fucose residues, which are absent in mammals. The immunogenicity and allergenicity of plant-specific $\mathrm{N}$ glycans is a key concern in mammalian therapy. In this study, we amplified the sequences of 2 plant-specific glycosyltransferases from Nicotiana tabacum L. cv Bright Yellow 2 (BY2), which is a well-established cell line widely used for the expression of therapeutic proteins. The expression of the endogenous xylosyltranferase (XyIT) and fucosyltransferase (FucT) was downregulated by using RNA interference (RNAi) strategy. The xylosylated and core fucosylated $\mathrm{N}$-glycans were significantly, but not completely, reduced in the glycoengineered lines. However, these RNAi-treated cell lines were stable and viable and did not exhibit any obvious phenotype. Therefore, this study may provide an effective and promising strategy to produce recombinant glycoproteins in BY2 cells with humanized N-glycoforms to avoid potential immunogenicity.
\end{abstract}

KEYWORDS BY2 cells, N-glycosylation, glycosyltransferase, RNA interference

\section{INTRODUCTION}

Plants have emerged as economical and convenient factories for large-scale production of recombinant therapeutic proteins (Twyman et al., 2003). Most pharmaceutical proteins are glycoproteins; $\mathrm{N}$-glycosylation of these proteins is essential for their solubility, stability, bioactivity, proper folding, and pharmacokinetics (Matsumoto et al., 1995; Dirnberger et al., 2001). Plants can perform N-glycosylation similar to mammals, which gives these plants a key advantage over other heterologous expression systems (Catherine Rayon, 1998; Lerouge et al., 1998). However, the process of glycosylation in plants and mammals varied greatly in the late Golgi apparatus (Gomord and Faye, 2004). This difference is mainly because of the residues in the core structure of plant complex N-glycans substituted by $\beta 1,2-x y l o s e ~(X y l)$ and $\alpha 1,3-$ fucose (Fuc), unlike $\alpha 1,6-F u c$ in mammalian $\mathrm{N}$-glycans. Moreover, plant $\mathrm{N}$-glycans are generally much smaller and lack the characteristic $\beta 1,4-$ galactose and sialic acid residues which were found in their mammalian counterparts. Consequently, the expression of mammalian glycoproteins in plants results in chimeric glycoprotein that have an $\mathrm{N}$-glycan profile mainly of complex $\mathrm{N}$-glycans containing $\beta 1,2-\mathrm{Xyl}$ and core $\alpha 1,3-F u c$ residues (Cabanes-Macheteau et al., 1999).

Although it has been previously reported that the absence of core a 1,6-Fuc may serve as an advantage for increasing Fc receptor $(F c R)$ binding and antibody-dependent cellular cytotoxic (ADCC) function of antibodies, the unwanted immunogenicity and allergenicity of the non-mammalian sugars $\beta 1,2-X y l$ and core $\alpha 1,3-$ Fuc cannot be excluded (van

"These authors contributed equally to this work. 
Ree et al., 2000; Niwa et al., 2004). These plant-specific Nglycans contain IgE binding carbohydrate determinants (Gomord et al., 2005). Furthermore, the production of $\beta 1,2-$ Xyl- and/or core- $\alpha 1,3-F u c-s p e c i f i c$ antibodies in goats, rabbits, and rats can be elicited by immunization with plant glycoproteins (Bardor et al., 2003). Hence, the immunogenicity of plant glycoepitopes remains a major concern in human therapy.

It has been documented that RNA interference (RNAi) is a valuable tool to eliminate the expression of core $\alpha 1,3-$ fucosyltransferase (FucT) and $\beta 1,2$-xylosyltransferase $(X y \mid T)$, which are enzymes responsible for the transfer of $\beta 1,2-\mathrm{Xyl}$ and core $\alpha 1,3-\mathrm{Fuc}$. This promising strategy effectively reduced the core $\mathrm{Xyl}$ and Fuc levels in Lemna minor (Cox et al., 2006). The glycoproteins synthesized in these RNAi-targeted lines carried predominantly humanized structures $(G n G n)$ devoid of plant-specific glycoepitopes.

The tobacco BY2 cell line has often been chosen as a wellcharacterized host for the production of exogenous proteins because of rapid growth rate, product purification and no contamination to environments (Fischer et al., 1999; Doran, 2000). In this study, tobacco BY2 cells (N. tabacum L. cV Bright Yellow 2) were engineered to achieve targeted downregulation of the expression of endogenous FucT and XyIT genes by using RNAi strategies. These glyco-optimized cell lines contained complex $\mathrm{N}$-glycan structures, but they strongly inhibited the incorporation of $\beta 1,2-X y \mid$ and core $\alpha 1,3-F u c$, which are responsible for the immunogenicity of plant $\mathrm{N}$-glycans, as determined by immunoblotting and matrix-assisted laser desorption-ionization time-of-flight mass spectrometry (MALDI-TOF MS).

\section{RESULTS}

\section{Cloning of $\beta 1,2-X y \mid T$ and $\alpha 1,3-F u c T$ cDNA fragments from tobacco BY2 cells}

To generate the glyco-engineered BY2 cell lines, we planned to use the gene knockout strategy for preventing the transference of plant-specific glycoepitopes. To achieve this, cDNAs encoding $\beta 1,2-X y \mid T$ and $\alpha 1,3-F u c T$ were isolated from tobacco BY2 cells and used as probes. First, cDNA sequences of XylT (EC 2.4.2.38) and FucT (EC 2.4.1.214) of several plant species were collected (http://www.cazy.org/), including Arabidopsis thaliana, N. tabacum, Oryza sativa, and Medicago truncatula; the conserved region was identified by aligning the sequences and degenerate primers were generated. Two DNA fragments, namely, NtXT (233 bp) and NtFT (933 bp) encoding $\beta 1,2-X y l T$ and core $\alpha 1,3-F u c T$, respectively, were amplified from tobacco BY2 cells using degenerate primers designed according to the homologous region sequences of these species. As predicted by NetPlantGene analysis, NtXT was a partial coding domain of $\beta 1,2-X y I T$ gene without an intron. Sequence similarity search with XyIT cDNA of other species was performed (http:// bioinfo.genopole-toulouse.prd.fr/multalin/). The NtXT amino acid sequence exhibited $98.3 \%$ and $67.0 \%$ similarity to $\alpha 1,2-$ XyIT from A. thaliana (At5g55500) and O. sativa (Os08 g0503800), respectively.

NetPlantGene analysis and sequence alignment with other plant FucTs revealed that NtFT contains an intron sequence from 194 to $854 \mathrm{bp}$. After splicing the intron domain, we performed sequence similarity search with FucT cDNA of other species (http://bioinfo.genopole-toulouse.prd.fr/multa$\mathrm{lin} /$ ). The NtFT amino acid sequence showed $75.3 \%$ and $77.1 \%$ identity with a1,3-FucT from A. thaliana (At3g19280, AtFucTA) and O. sativa (AP004457), respectively.

\section{Generation of glyco-engineered lines of tobacco BY2 cells}

To use the potential of tobacco BY2 cells for the production of recombinant pharmaceutical glycoproteins, it is highly desirable to prevent the incorporation of $\alpha 1,3-F u c-$ and $\beta 1,2-X y l-$ containing glycoepitopes to the proteins. This step is essential to prevent the immunogenicity of plant-specific $\mathrm{N}$ glycans in human therapy. To obtain a targeted downregulation of endogenous XylT and FucT gene expression, the amplified cDNA sequences of NtXT and NtFT were used to generate a binary RNAi construct. This construct, which was named FXR, contained the amplified 233 bp fragment of $\mathrm{XT}$ and $192 \mathrm{bp}$ fragment of FT cDNAs in the sense and antisense orientations, separated by an intron. The sense-intron-antisense hairpin RNA (ihpRNA) was supposed to target both the endogenous core $\alpha 1,3-F u c T$ and $\beta 1,2-X y l T$ of BY2 cells (Fig. 1C). BY2 transgenic lines were generated by Agrobacterium-mediated transformation, and the first screening based on kanamycin resistance was performed on a selection plate. Interestingly, all FXR lines were viable and exhibited no obvious morphological phenotype under standard growth conditions.

\section{Western blot analysis of glycoprotein xylosylation and fucosylation in FXR lines}

Independent transgenic XylT/FucT-RNAi lines (named as FXR1-12) were further screened by Western blot analysis to detect the presence or absence of the respective products: $\mathrm{N}$ glycans with $\mathrm{Xyl}$ and core $\alpha 1,3-\mathrm{Fuc}$ residues. Protein extracts from FXR lines were subjected to Western blot analyses using anti-horseradish peroxidase (anti-HRP) antibodies that recognize $\beta 1,2-X y l-$ and core $\alpha 1,3$-Fuc-containing structures. As shown in Fig. $2 \mathrm{~A}$, varying staining intensities were observed, indicating the presence of different amounts of $\mathrm{Xyl}$ and Fuc residues. Most of these knock-down transformants exhibited a large decrease in $\beta 1,2-x y l o s y l a t i o n$ and a1,3-fucosylation. One XylT/FucT-RNAi line (line 7; which was named FXR7), which exhibited very weak intensity after 
A

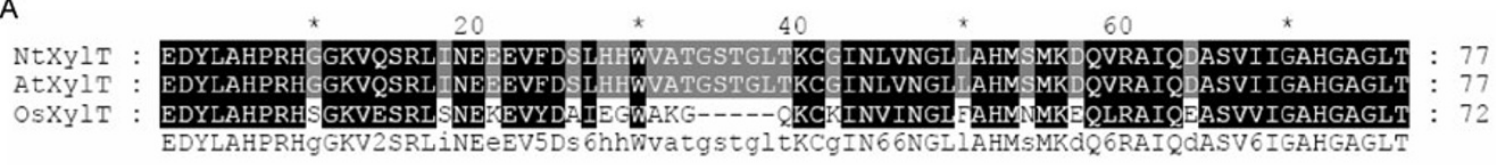

B

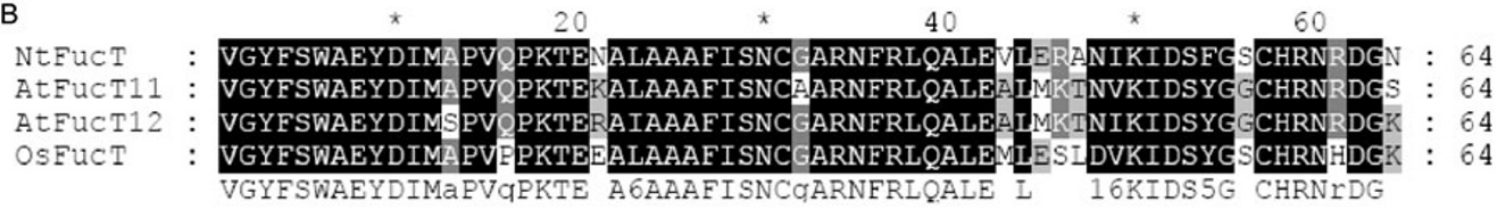

C

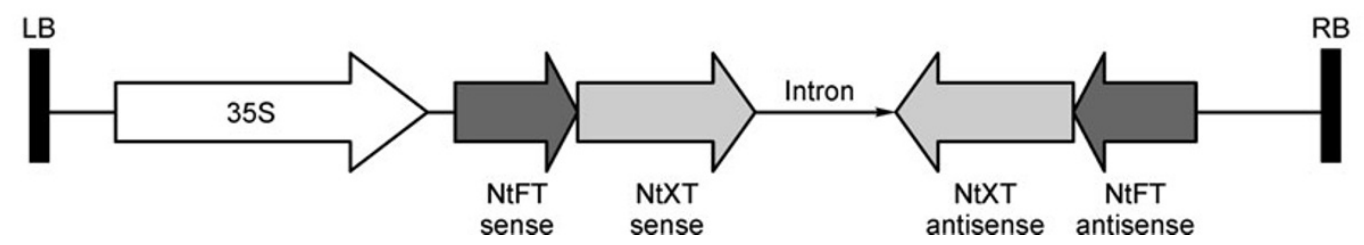

Figure 1. Mutiple sequence alignment and diagram of RNAi constructs. (A) Alignment of conserved motifs from $\beta 1,2-X y l T$ protein sequences. (B) Alignment of conserved motifs from a1,3-FucT protein sequences. The aligned amino acid sequences are from the plants Nicotiana tabacum L. cv Bright Yellow 2 (Nt), Arabidopsis thaliana (At) and Oryza sativa (Os). Black blocks represent amino acid identity between all sequences and gray blocks between two or three sequences. Broken lines correspond to the gaps required for the alignment of these sequences. (C) The construction diagram of FXR. The amplified DNAs encoding hairpin RNA (inpRNA) designed to target silencing of endogenous tobacco BY2 genes encoding $\alpha 1,3$-fucosyltransferase and $\beta 1,2-$ xylosyltransferase. RB and LB, right and left borders; 35S, cauliflower mosaic virus 35 S promoters.

staining with anti-HRP antibody, was identified and used for further analysis. It has been reported that cell growth influences the $\mathrm{N}$-glycosylation profile of endogenous proteins in tobacco BY2 cells (Elbers et al., 2001). Moreover, the levels of $\beta 1,2-X y l$ and $\alpha 1,3-F u c$ residues were elevated markedly in the late growth phase (from 5th to 7 th day after subculture) (Yin et al., 2009). Therefore, we determined the growth pattern of wild-type and FXR7 lines, and no obvious differences were observed between these 2 lines. Subsequently, the proteins from wild-type and FXR7 lines from the late growth phase were extracted and subjected to Western blot analyses using Xyl- and Fuc-specific antibodies, respectively. As compared to the wild-type cell line, the FXR7 line exhibited a marked decrease in $\beta 1,2-X y l-$ and core $\alpha 1,3-F u c-$ containing structures in the entire late growth phase (Fig. 2B). This observation indicated that rather than cell growth, inpRNA effectively eliminated the mRNA and significantly reduced the levels of $\beta 1,2-X y l$ and core $\alpha 1,3-F u c$ residues, thereby reducing the amount of endogenous glycosyltransferases.

\section{Structural analysis of N-glycans on total endogenous proteins from tobacco BY2 cell wild-type and RNAi lines}

To investigate the precise $\mathrm{N}$-glycoform profile of endogenous proteins, we subjected the wild-type and FXR7 lines of tobacco BY2 cells to MALDI-TOF MS. The total N-glycans were isolated from tobacco BY2 cells, as reported by Bakker et al. (2006). Briefly, the resulting N-glycan mixtures were analyzed by MALDI-TOF MS after digestion of total proteins by pepsin and peptide-N-glycosidase $A$. The mass spectrum showed a series of high-mannose-type $\mathrm{N}$-glycan ions ranging from Man5 to Man9 and complex-type N-glycans from a (MMXF) to e (GnGnXF) (Fig. 3). As expected, the mass spectrum revealed that the $\mathrm{N}$-glycan profile of wild-type tobacco cells had mainly complex-type $\mathrm{N}$-glycans bearing Xyl and $\alpha 1,3-F u c$ residues, including $\operatorname{MMXF}(a), \operatorname{GnMXF}(\mathrm{c})$, $\mathrm{Gn} 2 \mathrm{MX}(\mathrm{d})$, and GnGnXF (e). In contrast, the composition of $\mathrm{N}$-glycans in FXR7 lines revealed a remarkable decrease in plant-specific N-glycans. Although a small but significant amount of GnGnXF was still detected in FXR7, a major peak was identified as $\mathrm{GnGn}$, and the complex-type N-glycan without $\mathrm{Xyl}$ and Fuc residues was considerably increased and accounted for over $10 \%$ of the total oligosaccharides. It was worth noting that no N-glycan with a $\mathrm{GnGn}$ (b) structure was detected in wild-type cells. Further, in the XylT/FucT RNAi lines, some of complex-type $\mathrm{N}$-glycans did not contain the plant-specific residues and exhibited a sharp reduction in xylosylated and core fucosylated N-glycans (GnGnXF) because of incomplete downregulation of XylT and FucT activities. This result showed that the expression of an RNAi construct targeting the XylT and FucT in tobacco BY2 cells effectively prevented the accumulation of the plant-specific glycoepitope on complex N-glycan structure.

\section{DISCUSSION}

In this study, we investigated the feasibility of glycoengineering in tobacco BY2 cells. Partial cDNA sequences 
A

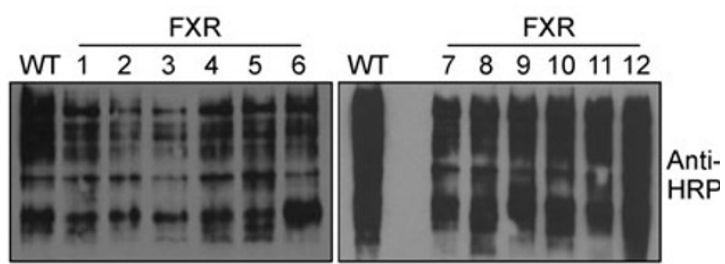

B

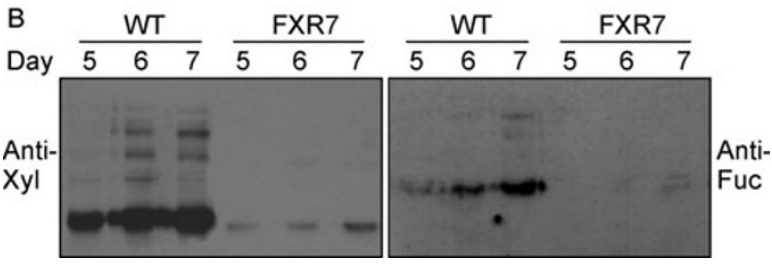

Figure 2. Analysis of the protein xylosylation and fucosylation in FXR lines. (A) Western blot analysis of total protein extracts from transgenic RNAi lines (FXR1-12). The presence/ absence of fucose and xylose residues was determined with anti-HRP antiserum. Numbers indicated the different transformed lines. (B) Western blot analysis of total protein extracts from WT and FXR7 lines from late growth phase using antixylose (left panel) and anti-fucose (right panel).

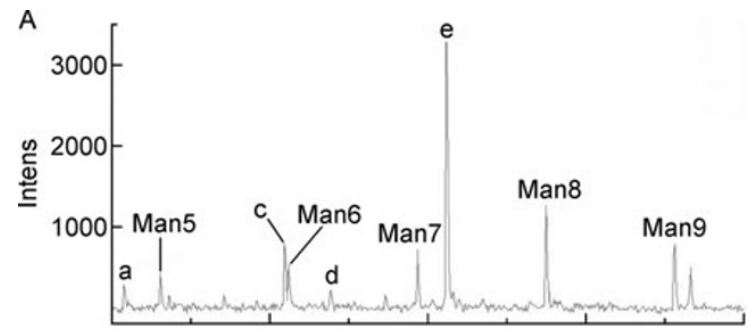

B

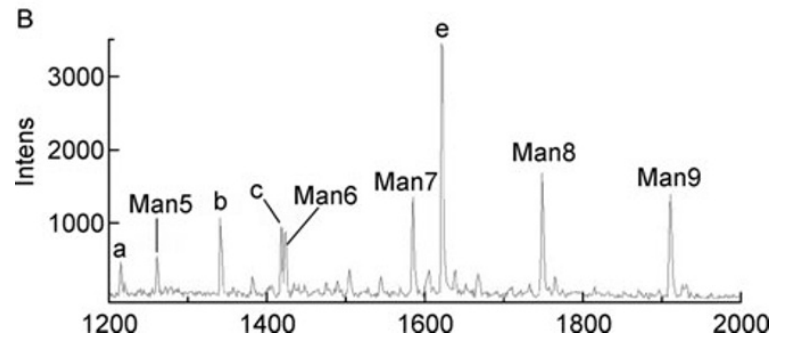

Figure 3. MALDI-TOF mass spectrum of $\mathrm{N}$-glycans isolated from endogenous proteins of WT (A) and FXR7 (B) cells.

of $\beta 1,2-\mathrm{XylT}$ and $\alpha 1,3-\mathrm{FucT}$ were cloned from tobacco BY2 cells. The sequences of these 2 proteins were used for generating RNAi construct for targeted down-regulation of endogenous XylT and FucT genes. The generation of glycoengineered transformants with a low level of $\beta 1,2-X y l$ and $\alpha 1,3-F u c$ residues was confirmed by Western blotting and MALDI-TOF MS.

Interestingly, XyIT/FucT k.o. plants of $A$. thaliana were viable and exhibited no obvious phenotype under standard growth conditions (Strasser et al., 2004). In addition, the transgenic plants (L. minor, N. benthamiana, and M. sativa), in which Xyl and Fuc residues were eliminated by RNAi, did not exhibit any obvious morphological phenotype (Cox et al., 2006; Sourrouille et al., 2008; Strasser et al., 2008). In our study, we also found that the deficiency of XylT and FucT did not affect the morphology, growth rate, or growth status of tobacco BY2 cells.

In previous studies, several strategies have been adopted to minimize the structural differences observed in complex $\mathrm{N}$ glycans of plants and mammals (Palacpac et al., 1999; Triguero et al., 2005). Cox et al. reported that RNAi was a valuable tool to eliminate the expression of core $\alpha 1,3-\mathrm{FucT}$ and $\beta 1,2-X y I T$ in the aquatic plant species $L$. minor (Cox et al., 2006). Surprisingly, this strategy was effectively applied to the production of a chimeric monoclonal antibody (mAb) that predominantly contained a single $\mathrm{N}$-glycan species $(\mathrm{GnGn})$ without any detectable plant-specific $\mathrm{N}$-glycans. More importantly, the glycan-optimized mAb exhibited high effector activities as compared to its Chinese hamster ovary (CHO)derived homologs. Recently, several studies also showed that RNAi effectively prevented the function of $\beta 1,2-X y \mid T$ and core $\alpha 1,3-F u c T$ in the whole plant of $N$. benthamiana and alfalfa, respectively (Sourrouille et al., 2008; Strasser et al., 2008). As compared to the whole plant, tobacco BY2 cells possess the merits of precise control over growth conditions, easy downstream processing, and no pollen contamination (Hellwig et al., 2004). However, little information is available about the 2 plant-specific glycosyltransferases in BY2 cells. According to the sequences of the conserved domain in several plant species, we designed degenerate primers to amplify the partial cDNA of endogenous $\beta 1,2-X y \mid T$ and core $\alpha 1,3$-FucT. The nucleotide sequences of these 2 amplified cDNAs exhibited high identity with XylT and FucT from $A$. thaliana and other plant species; furthermore, high sequence homologies were observed in the amino acid sequences of these glycosyltransferases. Previous studies have shown that $\beta 1,2-X y I T$ and core $\alpha 1,3-F u c T$ are type II membrane glycoproteins located in the membrane of Golgi apparatus (Wilson et al., 2001; Pagny et al., 2003). Similar to other glycosyltransferases, $\beta 1,2-X y l T$ and core $\alpha 1,3-F u c T$ contain a short $\mathrm{N}$-terminal cytosolic tail, a transmembrane domain, and a large luminal catalytic domain. Sequence analysis of the new amplified cDNAs in BY2 cells revealed that these structures were located in the conserved catalytic domain at the C-terminal end.

Western blot analysis revealed that the transgenic FXR lines exhibited a significant decrease in $\beta 1,2-X y l$ and core $\alpha 1,3-F u c$, although the $\mathrm{N}$-glycans of wild-type cells mainly contained these glycan residues. It has been reported that the developmental stage may influence the glycosylation profile of endogenous proteins in tobacco leaves and BY2 cells (Elbers et al., 2001; Yin et al., 2009). To exclude the effect of 


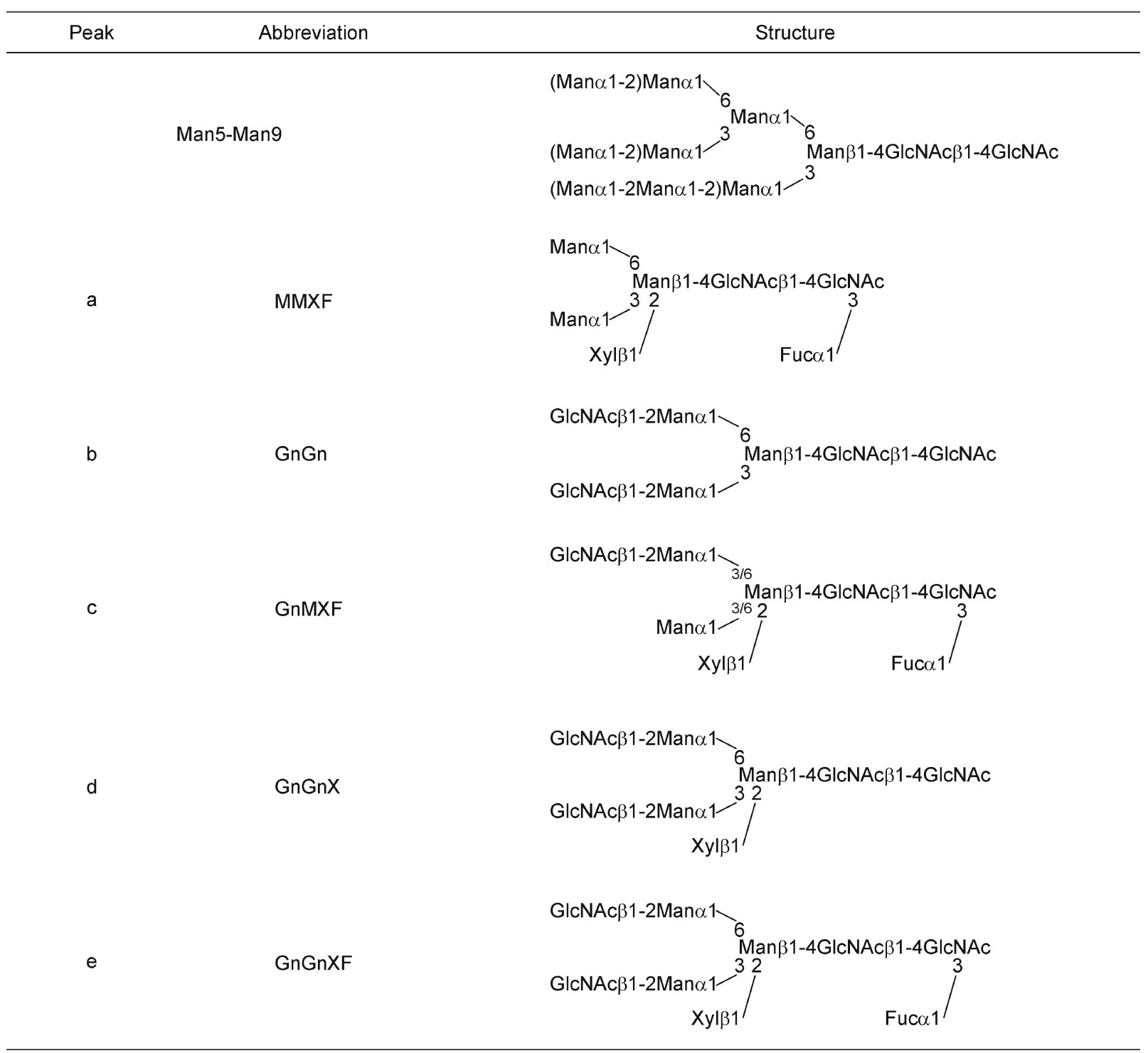

Figure 4. Structures of high-mannose-type (Man-5 to Man-9) and complex-type (a to e) $\mathrm{N}$-linked oligosaccharides extracted from wild-type BY2 cells.

cell growth on protein $\mathrm{N}$-glycosylation in this cell line, we determined its typical growth pattern. Interestingly, there was no influence of the status of cell growth on $\mathrm{N}$-glycosylation. To further evaluate the RNAi efficiency, we analyzed protein extracts from wild-type and FXR cells in the late growth phase (5th to 7th day) by Western blotting. The 2 plant-specific Nglycans were hardly detected during the entire growth cycle. Complete elimination of plant-specific oligosaccharides could not be achieved in the FXR7 line; however, the main epitope structure ( $\mathrm{GnGnXF}$ ) was present in only $21.4 \%$ of the total $\mathrm{N}$ glycans as compared to $45 \%$ of this structure in wild-type cells. Furthermore, an elevated level of GnGn structures devoid of plant-specific carbohydrate was detected by MALDI-TOF MS. The results of mass spectroscopy suggested that over $10 \%$ of total $\mathrm{N}$-glycans detected in the line
FXR7 had the GnGn structure that was not detected in the wild-type cells. This result suggests an obvious reduction of $\mathrm{Xyl}$ and Fuc residues in the RNAi lines despite incomplete downregulation of XylT and FucT activity. One explanation for this finding could be the presence of several copies of glycosyltransferases genes in tobacco BY2 cells. This observation has been described for other plant species. For example, 2 copies of a1,3-FucT (FucT A and FucT B) have been detected in Arabidopsis. Although there was no significant difference between FucT T-DNA insertion lines and wild-type line, the double-insertion line deficient in both FucT A and FucT B showed complete elimination of complextype $\mathrm{N}$-glycans with core $\alpha 1,3-\mathrm{Fuc}$ residues. It appears that both of FucT A and FucT B were active in vivo. In addition, there were no obvious changes in the other 2 minor peaks 
that were identified as MMXF and GnMXF, probably because of incomplete elimination of the 2 plant glycosyltransferases and the low level of MMXF and GnMXF.

Therefore, in this study, new partial cDNAs of XylT and FucT were amplified and the inhibition of the endogenous $\alpha 1,3-F u c T$ and $\beta-1,2-X y I T$ activities in glycol-optimized lines was accomplished by co-expression with a single RNAi transcript. The absence of plant-specific $\mathrm{N}$-glycans on the FXR transgenic BY2 line was confirmed by affinoblotting and MALDI-TOF MS. This strategy will pave the way to produce recombinant proteins devoid of plant glycol-epitopes in plant cells.

\section{MATERIALS AND METHODS}

Isolation of cDNAs encoding glycosyltransferases from tobacco BY2 cells

The core a1,3-FucT fragment was isolated from the cDNA of tobacco BY2 cells using the forward primer Fuc-Fw (5'GTTGGVTAYTTTTCRTGGG-3') and reverse primer Fuc-Rev (5'TCTGTDACATAATCYTCCTC-3'). Further, $\beta 1,2-X y I T$ sequence was amplified by polymerase chain reaction (PCR) using the primers XylFw (5'-GARGAYTACTTRGCCCATCC-3') and Xyl-Rev (5'-TGAGTYAGWCCWGCYCCATG- $3^{\prime}$ ). These 2 pairs of degenerate primers were designed based on the results of sequence homology analysis with annotated glycosyltransferases sequences and with known proteins from the database. The partial cDNA products were subjected to gel electrophoresis, purified, and then ligated into the pMD18-T vector (Takara) for sequencing.

\section{Construction of RNAi vectors for glycosyltransferases}

The PCR product of FucT (191 bp) was amplified using primers FucTS-Fw (CTCGAGACTAGTgttgggtattttcgtggg) and FucTS-Res (AAGCTTgggcatttccatcccggtt). Then, FucT was ligated into the Xhol/Hind III sites of the cloning vector psk-int to create psk-int-FI. The antisense fragment obtained by PCR using the primers FucTAFw (5'-TCTAGAACTAGTgttgggtatttcgtggg-3') and FucTA-Res (5'CCCGGGcatttccatcccggttac-3') was cloned into the Xbal/Smal site of the vector psk-int-FI to create psk-int-FR. The XylT fragment (233 bp) was then ligated into the Hind III sites of psk-int-FR, while the antisense XylT was cloned into the Smal site. Consequently, the "sense-intron-antisense" cassette was digested at the Spel site, and the product was cloned into the binary expression vector pCanG to create $p$ CanG-FXR. The plasmid pCanG-FXR was transferred into the Agrobacterium tumefaciens strain LBA4404 by electroporation.

\section{Cultivation of tobacco BY2 cells and transformation}

The tobacco BY2 cells (N. tabacum L. cv Bright Yellow 2) were subcultured on every 7 th day in Murashige and Skoog medium (Swiatek et al., 2002) (Sigma) of $\mathrm{pH} 5.8$, which contained 3\% (w/V)

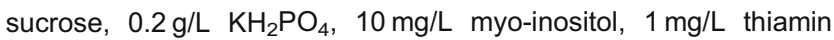
hydrochloride, and $0.2 \mathrm{mg} / \mathrm{L} 2,4-\mathrm{D}$. The cell line was regularly subcultured by transferring $1 \mathrm{~mL}$ of culture into $20 \mathrm{~mL}$ of fresh medium in a $100-\mathrm{mL}$ flask. The cultures were incubated in the dark at $26^{\circ} \mathrm{C}$ on a gyrating shaker at 120 rotation $/ \mathrm{min}$.
The tobacco BY2 cells were transformed by the Agrobacteriummediated method (Rempel and Nelson, 1995). Transgenic lines were selected and maintained in the abovementioned antibiotics-containing medium ( $250 \mathrm{mg} / \mathrm{L}$ carbenicillin sodium salt and $100 \mathrm{mg} / \mathrm{L}$ kanamycin).

\section{Glycoprotein analysis}

For N-glycans analysis, the total protein extracts from BY2 cells were prepared as described in the literature (Bakker et al., 2006). Tobacco cells were collected by centrifuge and then grinded in liquid nitrogen. The ground material was extracted in sodium dodecyl sulfatepolyacrylamide gel electrophoresis (SDS-PAGE) loading buffer containing $20 \mathrm{mM}$ Tris- $\mathrm{HCl}(\mathrm{pH} 6.8), 6 \%(\mathrm{v} / \mathrm{v})$ glycerol, $0.4 \%(\mathrm{v} / \mathrm{v})$ SDS, $20 \mathrm{mM}$ DTT, and $2.5 \mu \mathrm{g} / \mathrm{mL}$ bromophenol blue. The insoluble material was pelleted after incubation at $100^{\circ} \mathrm{C}$ for $5 \mathrm{~min}$. The supernatants $(12.5 \mu \mathrm{L}$ of each sample) were collected, subjected to $12 \%$ SDS-PAGE, and blotted to nitrocellulose membranes. Affinoblotting of $\beta 1,2$-xylosylated and/or $\alpha 1,3$-fucosylated proteins was performed with rabbit anti-HRP (Dingguo, Beijing), anti- $\beta 1,2-X y l$, and anti-a1,3-Fuc antibodies.

\section{$\mathrm{N}$-glycan purification}

The cultured BY2 cells were centrifuged, and total proteins were isolated from BY2 cells and $\mathrm{N}$-glycans were prepared as described (Yin et al., 2009).

\section{$\mathrm{N}$-glycan analysis}

The lyophilized $\mathrm{N}$-glycans were dissolved in $5 \mu \mathrm{L}$ of water per sample; $1 \mu \mathrm{L}$ of this solution was spotted on a stainless steel sample plate to which $1 \mu \mathrm{L}$ of S-DHB matrix (Sigma) was added, and this mixture was allowed to dry at room temperature. Positive-ion MALDI-TOF spectra were recorded on an Autoflex mass spectrometer (Bruker) fitted with delayed extraction and a nitrogen laser $(337 \mathrm{~nm})$. The spectra were generated from the sum of 200-300 laser pulses.

\section{ACKNOWLEDGEMENTS}

This work was supported by grants from National Natural Science Foundation of China (Grant No. 31030047) and the European Commission Sixth Framework Program (Grant No. SP22-CT-2004511060). We thank Ms. Shanting Hao for technical assistance.

\section{ABBREVIATIONS}

Fuc, fucose; FucT, fucosyltransferase; GlcNAc, N-acetyl-glucosamine; HRP, horseradish peroxidase; ihpRNA, sense intron-antisense hairpin RNA; MALDI-TOF, matrix-assisted laser desorption ionizationtime of flight mass spectrometry; Man, mannose; RNAi, RNA interference; SDS-PAGE, sodium dodecyl sulfate-polyacrylamide gel electrophoresis; Xyl, xylose; XylT, xylosyltransferase

\section{REFERENCES}

Bakker, H., Rouwendal, G.J., Karnoup, A.S., Florack, D.E., Stoopen, G.M., Helsper, J.P., van Ree, R., van Die, I., and Bosch, D. (2006). An antibody produced in tobacco expressing a hybrid beta-1,4galactosyltransferase is essentially devoid of plant carbohydrate epitopes. Proc Natl Acad Sci U S A 103, 7577-7582. 
Bardor, M., Faveeuw, C., Fitchette, A.C., Gilbert, D., Galas, L., Trottein, F., Faye, L., and Lerouge, P. (2003). Immunoreactivity in mammals of two typical plant glyco-epitopes, core alpha(1,3)fucose and core xylose. Glycobiology 13, 427-434.

Cabanes-Macheteau, M., Fitchette-Lainé, A.C., Loutelier-Bourhis, C., Lange, C., Vine, N.D., Ma, J.K., Lerouge, P., and Faye, L. (1999). $\mathrm{N}-$ Glycosylation of a mouse lgG expressed in transgenic tobacco plants. Glycobiology 9, 365-372.

Catherine, R., Patrice, L., Loic F. (1998). The protein N-glycosylation in plants. J Exp Bot 49, 1463-1472.

Cox, K.M., Sterling, J.D., Regan, J.T., Gasdaska, J.R., Frantz, K.K., Peele, C.G., Black, A., Passmore, D., Moldovan-Loomis, C., Srinivasan, M., et al. (2006). Glycan optimization of a human monoclonal antibody in the aquatic plant Lemna minor. Nat Biotechnol 24, 1591-1597.

Dirnberger, D., Steinkellner, H., Abdennebi, L., Remy, J.J., and van de Wiel, D. (2001). Secretion of biologically active glycoforms of bovine follicle stimulating hormone in plants. Eur J Biochem 268, 4570-4579.

Doran, P.M. (2000). Foreign protein production in plant tissue cultures. Curr Opin Biotechnol 11, 199-204.

Elbers, I.J., Stoopen, G.M., Bakker, H., Stevens, L.H., Bardor, M., Molthoff, J.W., Jordi, W.J., Bosch, D., and Lommen, A. (2001). Influence of growth conditions and developmental stage on $\mathrm{N}$ glycan heterogeneity of transgenic immunoglobulin $\mathrm{G}$ and endogenous proteins in tobacco leaves. Plant Physiol 126, 1314-1322.

Fischer, R., Emans, N., Schuster, F., Hellwig, S., and Drossard, J. (1999). Towards molecular farming in the future: using plant-cellsuspension cultures as bioreactors. Biotechnol Appl Biochem 30, 109-112.

Gomord, V., Chamberlain, P., Jefferis, R., and Faye, L. (2005). Biopharmaceutical production in plants: problems, solutions and opportunities. Trends Biotechnol 23, 559-565.

Gomord, V., and Faye, L. (2004). Posttranslational modification of therapeutic proteins in plants. Curr Opin Plant Biol 7, 171-181.

Hellwig, S., Drossard, J., Twyman, R.M., and Fischer, R. (2004). Plant cell cultures for the production of recombinant proteins. Nat Biotechnol 22, 1415-1422.

Lerouge, P., Cabanes-Macheteau, M., Rayon, C., Fischette-Lainé, A. C., Gomord, V., and Faye, L. (1998). N-glycoprotein biosynthesis in plants: recent developments and future trends. Plant Mol Biol 38, $31-48$.

Matsumoto, S., Ikura, K., Ueda, M., and Sasaki, R. (1995). Characterization of a human glycoprotein (erythropoietin) produced in cultured tobacco cells. Plant Mol Biol 27, 1163-1172.

Niwa, R., Hatanaka, S., Shoji-Hosaka, E., Sakurada, M., Kobayashi, Y., Uehara, A., Yokoi, H., Nakamura, K., and Shitara, K. (2004). Enhancement of the antibody-dependent cellular cytotoxicity of low-fucose IgG1 is independent of FcgammaRIlla functional polymorphism. Clin Cancer Res 10, 6248-6255.
Pagny, S., Bouissonnie, F., Sarkar, M., Follet-Gueye, M.L., Driouich, A., Schachter, H., Faye, L., and Gomord, V. (2003). Structural requirements for Arabidopsis beta1,2-xylosyltransferase activity and targeting to the Golgi. Plant J 33, 189-203.

Palacpac, N.Q., Yoshida, S., Sakai, H., Kimura, Y., Fujiyama, K., Yoshida, T., and Seki, T. (1999). Stable expression of human beta1,4-galactosyltransferase in plant cells modifies $\mathrm{N}$-linked glycosylation patterns. Proc Natl Acad Sci U S A 96, 4692- 4697.

Rempel, H.C., Nelson L. M. (1995). Analysis of conditions for Agrobacterium-mediated transformation of tobacco cells in suspension. Transgenic Res 4, 199-207.

Sourrouille, C., Marquet-Blouin, E., D'Aoust, M.A., Kiefer-Meyer, M. C., Seveno, M., Pagny-Salehabadi, S., Bardor, M., Durambur, G., Lerouge, P., Vezina, L., et al. (2008). Down-regulated expression of plant-specific glycoepitopes in alfalfa. Plant Biotechnol $\mathrm{J} 6$, 702-721.

Strasser, R., Altmann, F., Mach, L., Glössl, J., and Steinkellner, H. (2004). Generation of Arabidopsis thaliana plants with complex Nglycans lacking beta1,2-linked xylose and core alpha1,3-linked fucose. FEBS Lett 561, 132-136.

Strasser, R., Stadlmann, J., Schähs, M., Stiegler, G., Quendler, H., Mach, L., Glössl, J., Weterings, K., Pabst, M., and Steinkellner, H. (2008). Generation of glyco-engineered Nicotiana benthamiana for the production of monoclonal antibodies with a homogeneous human-like N-glycan structure. Plant Biotechnol J 6, 392-402.

Swiatek, A., Lenjou, M., Van Bockstaele, D., Inzé, D., and Van Onckelen, H. (2002). Differential effect of jasmonic acid and abscisic acid on cell cycle progression in tobacco BY-2 cells. Plant Physiol 128, 201-211.

Triguero, A., Cabrera, G., Cremata, J.A., Yuen, C.T., Wheeler, J., and Ramírez, N.I. (2005). Plant-derived mouse IgG monoclonal antibody fused to KDEL endoplasmic reticulum-retention signal is $\mathrm{N}$ glycosylated homogeneously throughout the plant with mostly high-mannose-type N-glycans. Plant Biotechnol J 3, 449 457.

Twyman, R.M., Stoger, E., Schillberg, S., Christou, P., and Fischer, R. (2003). Molecular farming in plants: host systems and expression technology. Trends Biotechnol 21, 570-578.

van Ree, R., Cabanes-Macheteau, M., Akkerdaas, J., Milazzo, J.P., Loutelier-Bourhis, C., Rayon, C., Villalba, M., Koppelman, S., Aalberse, R., Rodriguez, R., et al. (2000). Beta(1,2)-xylose and alpha(1,3)-fucose residues have a strong contribution in $\lg E$ binding to plant glycoallergens. J Biol Chem 275, 11451-11458.

Wilson, I.B., Rendić, D., Freilinger, A., Dumić, J., Altmann, F., Mucha, J., Müller, S., and Hauser, M.T. (2001). Cloning and expression of cDNAs encoding alpha1,3-fucosyltransferase homologues from Arabidopsis thaliana. Biochim Biophys Acta 1527, 88-96.

Yin, B., Zheng, N., Li, Y., Tang, S., Liang, L., and Xie, Q. (2009). Growth phase-dependent expression of proteins with decreased plant-specific $\mathrm{N}$-glycans and immunogenicity in tobacco BY2 cells. Sci China C Life Sci 52, 739-746. 